\title{
On the Cosmic Ray Sources
}

\author{
Guido Pizzella \\ Istituto Nazionale di Fisica Nucleare, Laboratori Nazionali di Frascati, Frascati, Italy \\ Email: guido.pizzella@Inf.infn.it
}

Received 16 January 2014; revised 15 February 2014; accepted 12 March 2014

Copyright (C) 2014 by author and Scientific Research Publishing Inc.

This work is licensed under the Creative Commons Attribution International License (CC BY).

http://creativecommons.org/licenses/by/4.0/

(c) (i) Open Access

\begin{abstract}
The cosmic ray energy spectrum is calculated assuming that cosmic rays are generated by astrophysical objects provided by magnetospheres with a dipole magnetic field. With simple geometric considerations, the energy spectrum $E^{-2.5}$ is obtained, independently on the particle species.
\end{abstract}

\section{Keywords}

\section{Cosmic Rays}

\section{Introduction}

Since the discovery of the cosmic radiation in 1912 by Victor Hess, the nature of the sources has been debated. After a century, mysteries still remain. Gold [1] suggested that cosmic rays be generated by the explosion of supernovae, but it is likely that there can be a variety of sources like, i.e. active galactic nuclei and, as suggested by Fermi [2], sources of continuos nature distributed in the intergalactic space.

As well known, in his original idea, Fermi considered an acceleration mechanism for cosmic rays based on the stochastic interaction of the charged particles available in space with wandering interstellar magnetic field clouds. He obtained for the accelerated particles a power law energy spectrum, and he stressed the importance to have obtained a power law, consistently with the experimental data. However, for explaining the value of the exponent in the experimental spectra, he needed to use certain values of the parameters regarding the particle energy threshold for this mechanism to operate and the energy losses in the interstellar space. We recall that the experimental differential energy spectrum is of the type

$$
\sim E^{\gamma}
$$

with $\gamma$ ranging $\sim-2.5,-3$ for all charged particles (protons, electrons, heavier particles).

At least another acceleration mechanism, acting locally, has been proposed in the past years [3]. The basic idea was the following. The source is an astrophysical body surrounded by charged particles in a strong 
magnetic field (i.e. pulsars, AGN, magnetospheric objects), constituting a magnetosphere ${ }^{1}$. In this region powerful acceleration mechanisms operate on the charged particles pushing them to go in the outer space as cosmic rays.

New recent observations seem to support this mechanism:

a) The result by Reeves et al. [4] concerning the Earth magnetosphere that quotes: The measurements show signatures of local acceleration by wave particle interactions in the heart of the radiation belts.

b)The observation [6] of ultrahigh-energy cosmic rays (UHECR), with energies above $6 \times 10^{19} \mathrm{eV}$, obtained with the Pierre Auger Observatory during 3.7 years, has shown that these cosmic rays have a non-isotropic spacial distribution. Quoting paper: The highest-energy particles originate from nearby extragalactic sources whose flux has not been substantially reduced by interaction with the cosmic background radiation. The Collaboration suggests that AGN or similar objects could be possible sources.

It is reasonable to think that if the Fermi theory applies also to the UHECR, one should expect an isotropic distribution for them, since the acceleration mechanism should operate everywhere in the intergalactic space. This is not verified by the Auger experiment.

These new experimental informations have pushed me to reconsider the magnetospheric mechanism with additional argumentations.

\section{The Magnetospheric Mechanism for the Cosmic Ray Sources}

Let us consider a celestial body with a magnetic field and a plasma atmosphere. We make the assumption that the magnetic field be generated by a dipole $\mathrm{M}$. The field at the equator at distance $r$ is given by

$$
B=\frac{M}{r^{3}}
$$

(For a neutron star with a radius $\sim 10 \mathrm{~km}$ the magnetic moment is of the order of $M=10^{21} \frac{\mathrm{T}}{\mathrm{m}^{3}}$.)

A charged particle with electrical charge $q$ and momentum $p$ can be trapped in a magnetic field with Larmor radius

$$
R_{L}=\frac{p}{q B}
$$

For a particle to stay trapped it is necessary that the Larmor radius be small enough to satisfy the Alfven condition [7]

$$
\frac{(\mathrm{d} B / \mathrm{d} r) R_{L}}{B} \leq \xi
$$

where the dimensionless quantity $\xi$ is estimated by means of plasma experiments in space and on the Earth and is of the order of a few per cent. Combining the above equations we get

$$
\frac{3 p r^{2}}{q M} \leq \xi
$$

This equation has to be interpreted in the following way: when an electrically charged particle accelerated by the electrical fields acting in the magnetosphere reaches a momentum p that violate Equation (5) (or it satisfies with the equal sign) then it escapes from the magnetosphere and goes into the outer space.

The plasma near the surface provides the source of the electrically charged particles as schematically shown in Figure 1. The accelerated particles follow the magnetic lines of force towards the equator, with a motion that obeys the laws of the adiabatic invariance [7]. At the equator the magnetic field is the weakest, so this is the place where it is more likely that the Alfven condition be violated.

We estimate now the energy spectrum of the escaping equatorial particles. At relativistic velocity the energy and momentum coincide. The number of particles contained in the magnetic shell between the lines of force reaching the equator at distances $\mathrm{L}$ and $\mathrm{L}+\mathrm{dL}$ (see Figure 1 ) is

\footnotetext{
${ }^{1}$ The observations of radiation belts of Jupiter and Saturn made with space probes stress the role of magnetospheres as sources of high energy particles. In some cases $\mathrm{MeV}$ electrons believed to be of Jovian origin have been observed in the vicinity of the Earth [5].
} 


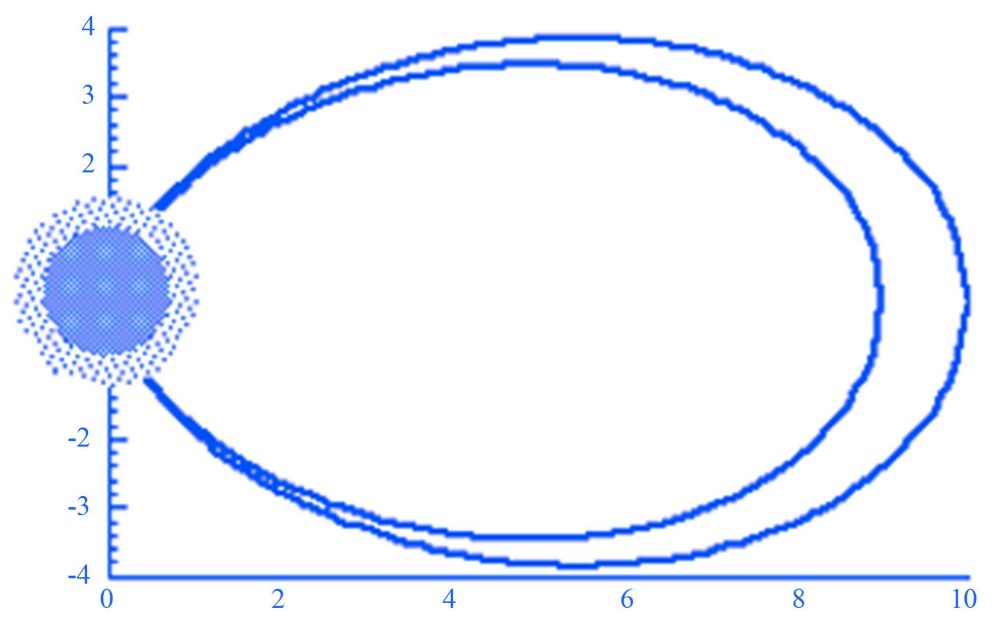

Figure 1. A pictorial view of the star with a dense plasma near its surface and two nearby lines of force of its dipole magnetic field.

$$
\mathrm{d} N \propto \rho(L) \mathrm{d} V
$$

where $\mathrm{d} V$ is the volume of the tridimensional shell delimited by the lines of force and $\rho(L)$ is the average particle density in the shell. For evaluating $\rho(L)$ we take into consideration the Liouville theorem. This theorem states that the density $\mathrm{N}$ of particles in a 6-dimensional space (position and momentum) is constant in a static $^{2}$ magnetic field along the particle trajectory.

Assume a plasma source with spherical distribution. Thus the density of the particles which are accelerated is, near the star surface, independent on the latitude. Each latitude corresponds to a distance $L$ at the equator according to the equation of the magnetic line of force

$$
r=L \sin ^{2}(\theta)
$$

where $\theta$ is the co-latitude. The particle flux $\mathrm{j}$ (number of particles per unit area, unit time and unit momentum) is constant along the particle trajectory (Liouville theorem), thus at the equator the particle flux is equal to that near the star surface, where the particle density is roughly independent on the latitude. This is equivalent to say that in Equation (6) we can consider $\rho(L)$ roughly independent on $\mathrm{L}$.

It remains to calculate the volume $\mathrm{dV}$ of the tridimensional shell delimited by the lines of forces corresponding to $\mathrm{L}$ and $\mathrm{L}+\mathrm{dL}$. We calculate

$$
\mathrm{d} V=\frac{64}{35} \pi L^{2} f\left(\frac{R}{L}\right) \mathrm{d} L
$$

where the function $f\left(\frac{R}{L}\right)$ tends to unity already for values of $L$ greater then twice the radius $\mathrm{R}$ of the solid
celestial body. Combining Equations (5) (with the = sign), (6) and (7) and putting $f\left(\frac{R}{L}\right)=1$ we get

$$
\mathrm{d} n \propto p^{-2.5} \mathrm{~d} p
$$

This is the number of particles with momentum between $\mathrm{p}$ and $p+\mathrm{d} p$ which leave, with relativistic velocity, the corresponding shell, since they satisfy Equation (4) with the equal sign.

We remark on the fact that a power law spectrum, with exponent very close to the experimental ones, has been obtained, on the basis of almost purely geometrical considerations. In the case of the Fermi's mechanism ad hoc values for the parameters involved in the initial acceleration as well in the particle loss in the interstellar space are necessary. Also we remark that the exponent $\gamma=-2.5$, obtained in a very simple way, is independent on the particle species, and compares favorably with the various measured spectra, all with power law distributions and exponent close to -2.5 .

\footnotetext{
${ }^{2}$ This is not exactly the case when electrical fields are acting, as it is necessary here for accelerating the particles. However the required acceleration mechanism is a very weak process; for the Earth magnetosphere the life time of $100 \mathrm{MeV}$ trapped protons is of the order of several years, such that small electrical fields are sufficient for the acceleration.
} 


\section{Final Remarks}

The above mechanism could be operative on small scales, like the planetary ones, or large scales as in the case of the cosmic rays proper. The maximum particle energy achievable with the above mechanism is obtained from the Equation (5)

$$
E_{\max }=\frac{\xi c q M}{3 r_{S}^{2}}
$$

where $r_{S}$ is the radius of the source and we take $\xi \sim 0.05$.

In the case of the Earth, with $r_{S} \sim 6400 \mathrm{~km}$ and $M=8 \cdot 10^{15} \frac{\mathrm{T}}{\mathrm{m}^{3}}$ we get
$E_{\max } \sim 980 \mathrm{MeV}$

which compares with the highest proton energies observed in the Van Allen belt.

In the case of Jupiter, with $r_{S} \sim 71500 \mathrm{~km}$ and $M=1.56 \cdot 10^{20} \frac{\mathrm{T}}{\mathrm{m}^{3}}$ we get

$$
E_{\max } \sim 15 \mathrm{GeV}
$$

We note that Jupiter could give a large contribution to the cosmic rays observed on the Earth surface with neutron monitors.

In the case of cosmic ray of extra solar-system origin, for a neutron star with $r_{S} \sim 10 \mathrm{~km}$ and $M=10^{21} \frac{\mathrm{T}}{\mathrm{m}^{3}}$, we calculate

$$
E_{\max }=5 \cdot 10^{19} \mathrm{eV}
$$

This value compares with the highest cosmic ray proton energy detected so far. Higher values can be obtained for larger values of $\mathrm{M}$.

The difference between the calculated value $\gamma=-2.5$ for the exponent of the power law spectrum and the experimental values, ranging roughly from -2.6 at low energy and -3 at higher energies, may be attributed to several causes; we mention: interaction of cosmic rays with the interstellar matter, acceleration by shocks from nova and supernova of different types [8], interaction with the microwave cosmic background [9], a non-dipole source mechanism. We can consider as cosmic ray sources not only neutron stars, but also various other collapsed objects with various radii and various strength of the magnetic field. A larger maximum energy can be accounted for in the case of the AGN, provided the dipole field model is still valid in such a case.

In all cases, we may propose that the Magnetospheric Acceleration Mechanism be the base for generating, for all types of particles, the power law energy spectrum.

\section{References}

[1] Gold, T. (1969) Nature, 221, 25. http://dx.doi.org/10.1038/221025a0

[2] Fermi, E. (1949) Physical Review, 75, 1169. http://dx.doi.org/10.1103/PhysRev.75.1169

[3] Pizzella, G. (1970) Nature, 226, 434. http://dx.doi.org/10.1038/226434a0

[4] Reeves, G.D., et al. (2013) Science, 341, 991-994. http://dx.doi.org/10.1126/science.1237743

[5] Teegarden, B.T., McDonald, F.B., Trainor, J.H., Webber, W.R. and Roelof, E.C. (1974) Journal of Geophysical Research, 79, 3615-3622.

[6] The Pierre Auger Collaboration (2007) Science, 318, 938.

[7] Alfven, H. and Falthammar, C.G. (1963) Cosmic Electrodynamics. Clarendom Press, Oxford.

[8] Zatsepin, V.I. and Sokolskaya, N.V. (2008) Astronomy \& Astrophysics, 5108.

[9] Anchordoqui, L.A., Dova, M.T., Epele, L.N. and Swain, J.D. (1998) Physical Review D, 57, 7103-7107. http://dx.doi.org/10.1103/PhysRevD.57.7103 